\title{
Managing residual risk in patients receiving statin therapy
}

\author{
Ian R Hamilton-Craig \\ Until the results of several statin trials are available, it is recommended that the current indications \\ and usage of ezetimibe be continued
}

$\mathrm{P}$ atients receiving statin therapy to reduce total and lowdensity lipoprotein cholesterol (LDL-C) levels still have a residual risk of cardiovascular $(\mathrm{CV})$ events. In most statin trials, CV events are reduced by about 30\% compared with placebo, leaving about $70 \%$ residual risk of $\mathrm{CV}$ events that occur in spite of statin therapy. Factors that may contribute to residual risk are listed in Box 1.

The Treating to New Targets Study ${ }^{1}$ showed that residual risk of $\mathrm{CV}$ disease increased with low levels of high-density lipoprotein cholesterol (HDL-C), even in patients who had low levels of LDL-C as a result of statin therapy. This suggested that raising HDL-C levels may be beneficial for such patients, a hypothesis investigated in the recent ARBITER 6-HALTS trial (ARBITER [Arterial Biology for the Investigation of the Treatment Effects of Reducing Cholesterol]; HALTS [HDL and LDL Treatment Strategies in Atherosclerosis]).

The ARBITER 6-HALTS trial was a study of carotid intima-media thickness (CIMT) in statin-treated patients who had achieved LDL$\mathrm{C}$ goal levels. It compared an HDL-C-raising strategy (additional treatment with extended-release nicotinic acid titrated to $2 \mathrm{~g}$ daily) with an LDL-C-lowering strategy (additional treatment with ezetimibe $10 \mathrm{mg}$ daily). ${ }^{2,3}$ The trial was stopped prematurely at its interim analysis of 180 of the planned 300 patients, on the basis of differences in the primary end point and other secondary analyses. ${ }^{4}$ The trial included subjects aged 30 years or over with coronary heart disease (CHD), known atherosclerotic vascular disease at other sites, diabetes, a 10-year CHD risk of $>20 \%$ (based on Framingham Heart Study criteria), or a coronary calcium score $>400$ in men or $>200$ in women, who were stabilised on statin therapy equivalent to simvastatin $20 \mathrm{mg} /$ day, with LDL-C levels $<2.5 \mathrm{mmol} / \mathrm{L}$ and HDL-C levels $<1.3 \mathrm{mmol} / \mathrm{L}$ in men or $<1.4 \mathrm{mmol} / \mathrm{L}$ in women. ${ }^{2}$ Exclusion criteria included raised transaminase levels more than three times the upper limit of normal, current use of or intolerance to ezetimibe or nicotinic acid, a history of chronic liver disease, or the potential for pregnancy.

Results of the ARBITER 6-HALTS trial showed that nicotinic acid reduced CIMT more effectively than ezetimibe (Box 2). Importantly, as no control group was included, no comparison can be made between ezetimibe and placebo with regard to their possible effects on CIMT.

Atherogenic dyslipidaemia may be an important contributor to residual risk and is typically associated with obesity, the metabolic syndrome and type 2 diabetes. It is accompanied by impaired glycaemic control, hypertension, and procoagulant and inflammatory states, and is characterised by low HDL-C levels, high triglyceride levels, the presence of triglyceride-rich "remnant" lipoproteins, and a preponderance of small, dense, highly-oxidisable LDL particles. Levels of total cholesterol and LDL-C may be close to normal.

Patients in the ARBITER 6-HALTS trial had a mean body mass index in the obese range $\left(30.8-31.0 \mathrm{~kg} / \mathrm{m}^{2}\right)$ and a waist circumference above normal (mean, $103 \mathrm{~cm}$ and $104 \mathrm{~cm}$ in the two groups); $32 \%-40 \%$ were diabetic; and $85 \%-86 \%$ were hypertensive. ${ }^{2}$ Mean glucose levels $(5.55-5.77 \mathrm{mmol} / \mathrm{L})$ and triglyceride
1 Possible contributors to residual risk in patients
receiving statin therapy
- Cigarette smoking
- Uncontrolled hypertension
- Impaired glucose tolerance
- Reduced physical exercise
- Central abdominal obesity
- Inflammation
- Prothrombotic tendency
- Proarrhythmic tendency
- Myocardial ischaemia
- Left ventricular dysfunction
- Reduced high-density lipoprotein cholesterol (HDL-C) level
- Increased low-density lipoprotein cholesterol (LDL-C) level
- Atherogenic dyslipidaemia:
$>$ Increased apolipoprotein B and small, dense LDL particles
$>$ Reduced HDL-C level
> Increased level of triglycerides and triglyceride-rich lipoproteins

levels (1.38-1.42 mmol/L) were also in the high-normal range. Patients were selected for inclusion in the trial on the basis of low HDL-C levels. HDL-C levels increased, as expected, in the nicotinic acid treatment group (by about 18\%), but unexpectedly decreased in the ezetimibe treatment group (by about $5 \%$ ). ${ }^{2}$ The results of the trial may thus be partially explained by the fact that

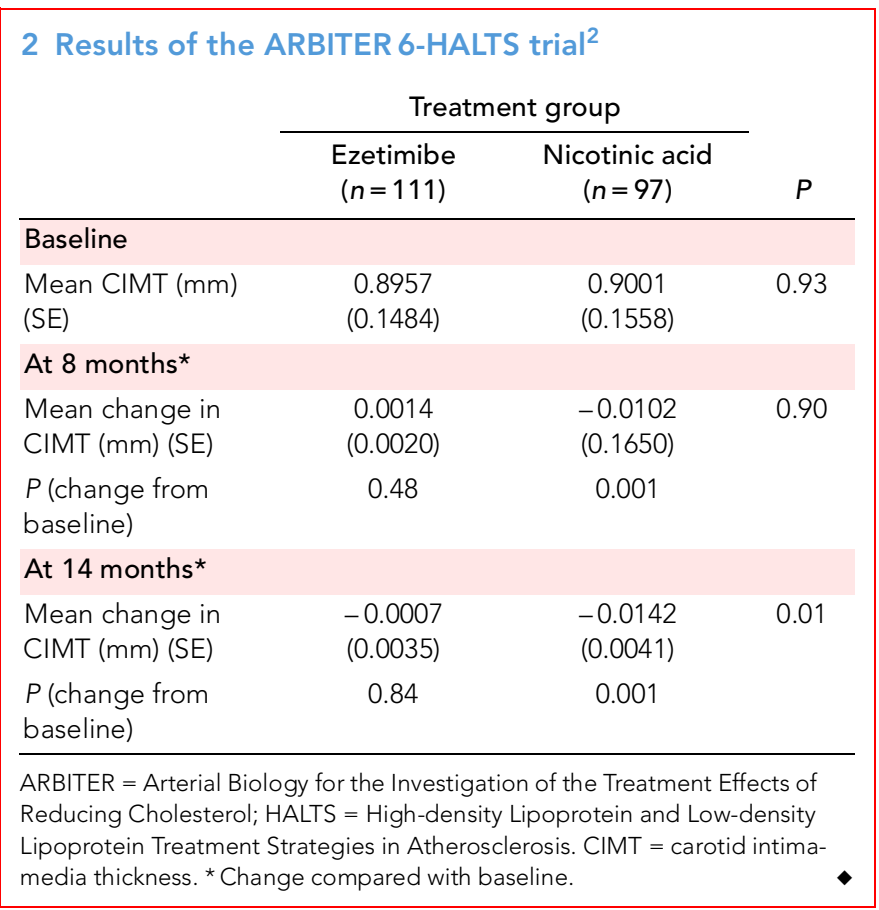




\section{Summary and future prospects}

The ARBITER 6-HALTS trial showed that a strategy of raising highdensity lipoprotein cholesterol (HDL-C) levels using nicotinic acid therapy was more successful than a strategy of lowering low-density lipoprotein cholesterol (LDL-C) levels further with ezetimibe, at least with regard to reducing carotid intima-media thickness.

Results of the trial are relevant to patients receiving statin therapy who have LDL-C levels $<2.5 \mathrm{mmol} / \mathrm{L}$. Many of these patients have atherogenic dyslipidaemia, with low levels of HDL-C and high levels of triglycerides, as well as increased levels of apolipoprotein B and small dense atherogenic LDL particles. Despite having statin therapy, such patients may continue to experience a high rate of cardiovascular disease events - in part because of lack of control of atherogenic dyslipidaemia, which is not targeted by statins or ezetimibe but is alleviated by fibrates and nicotinic acid. The demonstrated superiority of nicotinic acid therapy in the ARBITER 6HALTS trial is probably the result of improved control of atherogenic dyslipidaemia.

Although the results of the trial are potentially important, their relevance to Australian practice is limited because extended-release nicotinic acid, although approved for prescription by the

Therapeutic Goods Administration, is not reimbursed by the Pharmaceutical Benefits Scheme. The few patients who can tolerate non-extended-release nicotinic acid at doses of $2 \mathrm{~g}$ daily may benefit from this agent when added to statin therapy. An alternative strategy may be to combine statin therapy with fenofibrate therapy (the effectiveness of this approach will be determined by the results of the ACCORD trial, to be presented in early 2010). Other trials are in progress to determine the long-term clinical outcomes of statin therapy combined with ezetimibe (IMPROVE-IT [estimated completion date, 2015]) and with extended-release nicotinic acid (AIM HIGH and HPS2-THRIVE [estimated completion dates, 2011 and 2013, respectively]).

Pending the results of these trials, the continued use of ezetimibe is recommended for patients unable to tolerate statins and for those whose LDL-C level is uncontrolled in spite of maximum-tolerated doses of statins.

ACCORD = Action to Control Cardiovascular Risk in Diabetes. AIM HIGH = Atherothrombosis Intervention in Metabolic Syndrome with Low HDL/High Triglycerides and Impact on Global Health Outcomes. ARBITER = Arterial Biology for the Investigation of the Treatment Effects of Reducing Cholesterol; HALTS $=\mathrm{HDL}$ and LDL Treatment Strategies in Atherosclerosis. HPS2-THRIVE = Heart Protection Study 2 Treatment of HDL to Reduce the Incidence of Vascular Events. IMPROVE-IT = Improved Reduction of Outcomes: Vytorin Efficacy International Trial.

a significant proportion of patients had atherogenic dyslipidaemia, which responds to nicotinic acid therapy but not to ezetimibe therapy.

Conclusions from the ARBITER 6-HALTS trial about the LDL-Clowering effects of ezetimibe and the HDL-C-raising effects of nicotinic acid may be questioned, as ezetimibe and nicotinic acid affect different lipoproteins and metabolic pathways that may influence atherosclerosis. Nicotinic acid lowers levels of triglycerides and triglyceride-rich lipoproteins by reducing free fatty acid flux to the liver; it also lowers lipoprotein (a) and LDL-C levels. Ezetimibe has less pronounced effects on lowering triglyceride levels and raising HDL-C levels.

In addition, the protective functions of HDL-C (including reverse cholesterol transport, anti-inflammatory and antioxidant effects) may not necessarily be reflected in HDL-C levels, and functional assessment of HDL-C may assist in assessing the potential benefits of intervention. ${ }^{5}$
The implication of the ARBITER 6-HALTS trial results is that targeting atherogenic dyslipidaemia with nicotinic acid therapy reduces CIMT and is likely to improve residual risk of CV events because CIMT is a validated surrogate marker for CV events - an important consideration for all patients receiving statins. ${ }^{6}$ The HATS (HDL-Atherosclerosis Treatment Study) trial demonstrated that CV events could be reduced by treating atherogenic dyslipidaemia with nicotinic acid. ${ }^{7}$ Previous CIMT studies (ARBITER 2 and 3) showed that a combination of nicotinic acid and statin therapy was superior to statin therapy alone for atherosclerosis stabilisation and regression. ${ }^{8}$ These results, together with those of the ARBITER 6-HALTS trial, indicate that nicotinic acid may be appropriate supplementary therapy for reducing residual risk in patients taking statins. The results also provide an evidence base for extended-release nicotinic acid to be made available once again under the Pharmaceutical Benefits Scheme.

Long-term clinical outcomes trials are already underway to investigate the primary findings of the ARBITER 6-HALTS trial (Box 3). ${ }^{2}$ Until the results of these trials are available, it is recommended that the current indications and usage of ezetimibe be continued (Box 3).

Lifestyle change is an important component of managing residual risk (Box 1). Stopping smoking, controlling weight and increasing physical exercise can all increase HDL-C levels and may contribute independently to reduction in residual risk of $\mathrm{CV}$ events.

\section{Competing interests}

Ian Hamilton-Craig has received financial support to attend and give presentations at scientific meetings from AstraZeneca, Merck Sharp \&amp; Dohme, Schering-Plough, Pfizer, Bristol-Myers Squibb, Solvay, SanofiAventis, Novartis and Abbott Australasia. He is a member of the Lipid Advisory Board of Merck Sharp \&amp; Dohme/Schering-Plough, AstraZeneca and Solvay, the Familial Hypercholesterolaemia Committee of the Australian Atherosclerosis Society, and the Council on Genetic Cardiovascular Diseases of the Cardiac Society of Australia and New Zealand.

\section{Author details}

Ian R Hamilton-Craig, MB BS, FRACP, PhD, Professor of Preventive

Cardiology

School of Medicine, Griffith University, Gold Coast, QLD.

Correspondence: i.hamilton-craig@griffith.edu.au

\section{References}

1 Barter P, Gotto AM, La Rosa JC, et al. HDL cholesterol, very low levels of LDL cholesterol, and cardiovascular events. N Engl J Med 2007; 357: 1301-1310.

2 Taylor AJ, Villines TC, Stanek EJ, et al. Extended-release niacin or ezetimibe and carotid intima-media thickness. N Engl J Med 2009; 361: 2113-2122.

3 Kastelein JJ, Bots ML. Statin therapy with ezetimibe or niacin in high-risk patients. N Engl J Med 2009; 361: 2180-2183.

4 Blumenthal RS, Michos ED. The HALTS trials - halting atherosclerosis or halted too early? N Engl J Med 2009; 361: 2178-2180.

5 Sorrentino SA, Besler C, Rohrer L, et al. Endothelial-vasoprotective effects of high-density lipoprotein are impaired in patients with type 2 diabetes mellitus but are improved after extended-release niacin therapy. Circulation 2009; 121:110-122.

6 Hodis HN, Mack WJ, LaBree L, et al. The role of carotid intima-media thickness in predicting clinical coronary events. Ann Intern Med 1998; 128: 262-269.

7 Matthan NR, Giovanni A, Schaefer EJ, et al. Impact of simvastatin, niacin, and/or antioxidants on cholesterol metabolism in CAD patients with low HDL. J Lipid Res 2003; 44: 800-806.

8 Taylor AJ, Lee HJ, Sullenberger LE. The effect of 24 months of combination statin and extended-release niacin on carotid intima-media thickness: ARBITER 3. Curr Med Res Opin 2006; 22: 2243-2250. 